\title{
$\operatorname{CUNF}-8408123--2$
}

CONF-8408123--2

DE86 002014

\section{SUMMARY REMARKS AND FUTURE PROSPECTS FOR ON-LINE NUCLEAR ORIENTATION}

\author{
K. S. Krane \\ Physics Department \\ Oregon State University \\ Corvallis; OR 97331
}

\author{
J. H. Hamilton \\ Physics Department \\ Vanderbilt University \\ Nashville, TN 37235
}

\section{International Symposium on Nuclear Orientation and Nuclei far from Stability \\ Leuven, Belgium \\ August 28-31, 1984 \\ A505- 76ERCS0.34}

\section{DISCLAIMER}

This report was prepared as an account of work sponsored by an agency of the United States Government. Neither the United States Government nor any agency thereof, nor any of their employees, makes any warranty, express or implied, or assumes any legal liability or responsibility for the accuracy, completeness, or usefulness of any information, apparatus, product, or process disclosed, or represents that its use would not infringe privately owned rights. Reference herein to any specific commercial product, process, or service by trade name, trademark, manufacturer, or otherwise does not necessarily constitute or imply its endorsement, recommendation, or favoring by the United States Government or any agency thereot. The views and opinions of authors expressed herein do not necessarily state or reflect those of the Unitei States Government or any agency thereof. 
SUMMARY REMARKS AND FUTURE PROSPECTS FOR ON-LINE NUCLEAR ORIENTATION

K. S. KRANE* and J. H. HAMILTON**

"Physies Department, Oregon State University, Corvaliis, Oregon, USA 97331

*Physics Department, Vanderbilt University, Nashvilie, Tennessee, USA 37235

1. Introduction

Nuclear orientation (NO), like other suis-specialty fields including perturbed angular correlations and Mossbauer effect, has existed for many years as a basic technique for studying hyperfine interactions, which is often rather loosely defined as the interaction of nuclei with their environment. These techniques. thus have the potential to elucidate problems in nuclear physics and in solid-state physics. Indeed, early low temperature NO work in the mid-1950's revealed many new properties of paramagnetic salts and also established many nuclear spectroscopic parameters, including spins, moments, and radiation multipolarities of certain relatively simple and long-lived radioactive decay schemes such as ${ }^{60} \mathrm{Co}$ and ${ }^{54 \mathrm{Mn}}$; (A review of the development of the field is given by Postma and Stone [1].) In the late 1950's two important advances occurred: the parity-violating ${ }^{6}$ Co beta-decay experinent of Wu et al. [2], which showed that NO could be used to attack nuclear problems of fundamentai interest, and the discovery that ferromagnetic metals could be used as host lattices for NO, which greatly extended the range of orientable nuciei by replacing the need to incorporte them chemically into a paramagnetic salt with the need to introduce them physically as a component of a dilute alloy.

The late 1960's saw two further technological developments--the dilution refrigerator and the $\mathrm{Ge}(\mathrm{Li})$ detector--that boosted the capability of NO as a nuclear spectroscopic tool. The No investigator could now study decay schemes at high resolution and could do so for long periods, permitting even very weak transitions to be precisely measured. For more than a decade, NO laboratories continued to turn out precise and useful nuclear spectroscopy data, refining, elaborating, and often correcting previous knowledge, but it is clear that this work did not reveal any new or unexpected features of nuclear structure and that the frontiers of research of low-energy nuclear physics lay elsewhere.

One such frontier is the study of the short-lived decays of nuclei far from stability. Following the development of isotope separators on-line with accelerators and reactors over a decade ago, studies of nuclei far from stability have provided many new insights and led to numerous discoveries in the shapes, motions and structure of nuclei. These discoveries include, for example, the coexistence of both near-spherical and deformed shapes in one nucleus, unexpected new egions of very strong deformation, the importance of reinforcing shell gaps for both protons and neutrons on the nuclear shape, and the importance of new sheil gaps at different proton and neutron numbers on the structure of nuclei. There are other important discoveries in this field which do not bear on No studies, such as proton radioactivity of nuclear ground states, beta-delayed twoand three-neutron decay and beta-delayed two-proton decay. The importance of these discoveries and the broad ranges of unexplored nuclear terrain still farther from stability have made this field a major frontier in nuclear science (see reviews by Hansen [3] and Hamilton et al. [4]). Now, as we will show, nuclear orientation facilities on-line with on-line separators can have very significant impacts on the quality and quantity of data in unexplored regions 
far from stability. More important, such systems likely will provide the only means, in many cases, of extracting data which are essential to test different nuclear models. Thus, in the future we expect dilution refrigerators on line with isotope separators to assume an ever increasing role in nuclear structure studies far from stability.

Let us look briefly at why we assert that on-line NO experiments will begin to play a fundamental role in exploring nuclear structure far from stability. Within a few nucleons of the "valley of stability," radioactive decays are long-lived ( $t_{1 / 2}$ : hours or days), giving the experimenter reasonable time to gather the required data. Moreover, in this region the states can usually be populated directiy through few-particle transfer reactions tiles $(A \leq 4)$; frequently by choosing both low-spin and high-spin stable targets to reach $\bar{a}$ given nuclide, one $c$ an populate nearly all of the ievels of interest. Few-particle transfer reactions tend to be "clean" in the sense that it is of ten possible to reduce backgrounds by a careful selection of the incident energy to maximize the cross-section for a particular set of products.

As one climbs out of the valley of stability, this happy situation no longer occurs. The decay lifetimes become so short $\left(t_{1 / 2}-\right.$ minutes or

seconds) that conventional off-line techniques are not practical. The only nuclear reactions that can reach these profucts for in-bean spectroscopy are those involving relatively heavy projectiles. With relatively high energies needed to overcome the Coulomb barrier, a multitude of products usually results. Moreover, these reactions tend to transfer large angular momenta to the product nuclei, and thus a rather limited set of states (those close to the yrast line) is populated. Close to the stable nuclei, the beta decay energies are small, of the order of 0.5 to $1.5 \mathrm{MeV}$, so that often only a very few excited states are populated. In this region nuclear reations are frequently far more useful in elucidating the level structure. Far from stability, on the other hand, radioactive decays with their large decay energies, typically 3-6 MeV, have the advantage of populating a rich spectrum of excited states including collective and single particle structures. In addition, in odd-Z, odd-N nuclei there are frequently two beta-decaying isomers, one with low spin and one with high spin, and so the decays can reach many different excited configurations.

Angular distributions of radiations enitted by oriented nuclei are essential in determining spin assignments and radiation multipolarities. Nuclear reactions provide a means of orienting the product nuclei, but (as has bean argued abovel are only of limited value in studying nuclei far from stability. Angular correlations of successive radiations can do likewise, but suffer from the reduced intensities of coincidence measurements. Low-temperature nuclear orientation of the radioactively decaying nuclei suffers from neither of these problems and hence has the potential to contribute detailed and systematic knowledge about nuclear structure.

\section{On-line nuclear orientation and nuclear spectroscopy}

The technological development that has made on-line NO possible is the top-loading, side-access dilution refrigerator [5]. Already, there are three operating facilities in which an isotope separator is on-line to a dilution refrigerator, permitting relatively short-lived nuclei to be directly implanted into an orienting environment. These are in Bonn [6], Leuven [7], and Daresbury [8]. At least two additional facilities (Dubna and UNISOR) are in the planning and construction stage. The technological details of these facilities, including the accelerator and isotope separator, need not be reviewed here. Rather, we will address the critical problem that has been studies in many of 
the experiments reported thus $f a r:$ what is the nature of the site seen by an

implanted ion and what hyperfine field (or distribution of fields) does it experience?

Because ordinary angular distribution experiments average over the field distribution, they are not able to deal with this question in any detail. It is necessary to use nuclear magnet ic resonance on or iented nuclei (NMR/ON), which has the capability to isolate specific field sites and give their relative populations. Room-temperature implantation has for many years been used to prepare samples for NO work, and for PAC and Mossbauer experiments as well. The effect of the implantation in producing "good" substitutional sites has been thoroughly studied, and the effects of radiation damage on the environment of the implanted atom are at least documented, if not well understood. What is less clear is the effect of room temperature annealing on the population of these "good" sites and what the result will be when implanting into an environment at $T<.1 K$.

The first group to report results from on-line implartation at $T<1 K$ was the Bonn group [6], in which the refrigerator is on-line to an isotope separator but not to an accelerator, so that only relatively long-lived (- hours) decays can be studied. This group has carried out a careful and systematic study of implantation behavior using the NMR/ON technique. The first reported cold

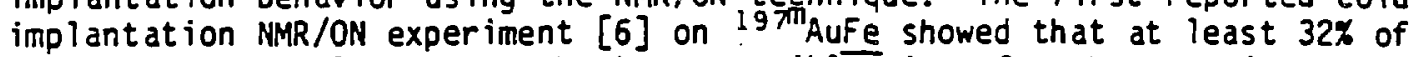
the implanted nuclei occupy the best possible sites for the experimenter--substitutional sites without radiation damage. Moreover, the NMR linewidth was substantially smaller in the cold implantation sample than it was in a similar study using room-temperature implantation; this likewise suggests undamaged sites. The Bonn group has also studied NMR/ON in the system $206_{B}$ ife; bismuth is insoluble in iron and no resonance for this system had been previousily reported. A rather broad resonance signal was found [9] which was interpreted as having two components, a narrow line at the expected resonant frequency and a very broad line at a slightly higier frequency. Assuming the narrow line to represent substitutional nuclei in good sites, the Bife hyperfine field is deduced to be in excellent agreenent with the value found in a nonresonanc No experiment. In two experiments reported at this symposium (by Herzog et al.) a similar combination of narrow and broad lines was found for cold implanted ${ }^{206} \mathrm{BiNi}$ and a single rather broad line was found for $177_{\text {LuFe; the former }}$ resonance yielded a hyperfine field in good agreement with that of a static NO experiment, while the latter gave a hyperfine field nearly $20 \%$ larger than the static NO result.

Based on these results, we may draw a number of conclusions regarding the potential of on-line NO for nuclear spectroscopy: (1) Cold implantation gives a sufficient population of "good" hyperfine field sites. (2) NMR is essential if one wishes unambiguously to measure the spin of the decaying partont or its magnetic moment. (3) Comparisons between static and resonant No experiments must be done with caution. (4) Nuclear spectroscopic conclusions shou id generally not be affected by the uncertainties in hyperfine structure, since one usually calibrates with a transition of known parameters in the decay scheme.

The first reported on-line experiments with short lived nuclides, with the isotope separator and refrigerator on-line to an accelerator as well, are those of the Leuven group [10], in which the magnetic moments of 105-i08in were determined by nonresonant NO. All of these activities have-halflives of less than one hour, the shortest being five minutes. Coupled with results of other studies (including previous off-line work by the sane group) it is possible to trace the influence of the $g_{9 / 2}$ proton on the magnetic moments of states of In from $A=105$ to $A=116$, including several odd $-Z$, odd $-N$ isotopes is which there are two or more isomers, representing different couplings with the $g_{9 / 2}$ proton. A later work presented similar results for $101,102 \mathrm{Ag}[11]$. 
It might be argued that these results from nonresonant experiments are somewhat anbiguous in the light of the previous discussion of the uncertainties in implantation behavior. However, the problem was avoided by simultaneously implanting an isotope of known magnetic moment $\left(110 \mathrm{~m}\right.$ In and $10{ }^{3} \mathrm{Ag}$, respectively), and the comparison of the hyperfine structures permits the reliable deduction of the unknown magnetic moments.

The Leuven group has also studied the effects of cold implantation. First, in the case of room temperature implantation, they observed [12] the beta anisotropy of $1{ }^{14}$ Infe. Nonresonant data yielded a slightly smaller moment than the NMR result, suggesting that some of the implanted nuclei (but less than 18\%) were not in good substitutional sites. Cold implantation of llin (off-line) and $103 \mathrm{Ag}$ into $\mathrm{Fe}[13]$ showed essentially $100 \%$ in good substitutional sites by using nonresonant $\mathrm{NO}$, if the implantation dose is kept sufficiently low that an implanted atom has a negligible probability to experience a radiation-damaged environment created by another implanted ion. As Nuytten et al. point out [13], their results are certainly encouraging for on-line studies far from stability, even if NMR is not available. Consistent results were obtained [14] upon implantation into $C_{0}:$ room-temperature implantation gives $=40 \%$ in good sites, while cold implantation gives essentially 100\%.

Recent on-line measurements by the Leuven group include NMR/ON on ${ }^{106}$ In and $104 \mathrm{Ag}$ as well as nuclear spectroscopic studies of the decays of $185,186,189,191_{A u}$ to states in Pt, reported at this Symposium (see Vandeplassche et al., van Walle et al., Wouters et al.).

The Daresbury group have now rérorted here their first on-line No results (see Green et al.). They observed nonresonant NO on implanted $118,120 \mathrm{I}$ and ${ }^{20} \mathrm{Cs}$ in Fe. $\bar{A}$ large fraction $(-80 \%)$ of the I nuclei were deduced to occupy good substitutional sites, and magnetic moments of the I isomers were deduced. The $C s$ orientation is the first reported for any $C_{S}$ isotope, and the two $120 \mathrm{Cs}$ isomers represent the shortest-lived nuclei yet oriented by direct implartation (64s for the low-spin isomer and $57 \mathrm{~s}$ for the high-spin). In fact, it is likely that the spin-lattice relaxation time $T_{1}$ for the high-spin isomer is roughiy the same as its half-life; thus the ${ }^{120} \mathrm{Cs}$ is incompletely oriented and the analysis of the observed anisotropies becomes difficult. A particular goal of these studies was the deduction of the 118, $120 \mathrm{Te}$ level schemes and their comparison with calculations based on the IBA-II model.

Up to now we have discussed specifically the on-line NO experiments reported at this Symposium. Many other papers have been presented which have direct and substantial impact on the potential to do nuclear spectroscopy far from stability using NO. In the area of technology, we have learned of the continuing development of the beam-foil and bean-surface methods of nuclear orientation (see, for example, the Symposium papers of Winter and of Deutsch). Such methods will surely be important when we try to study nuclei so short-lived that they have not sufficient time to relax into equilibrium with the implanted environment. In addition laser NO may possibiy play a role in this area (see J. Bonn). We have also learned of the possibility (see Eska) of a nuclear demagnetization stage being able to maintain temperatures at or below 1 mK for a time of the order of one day.

Angular correlations are an alternative to NO for decay scheme studies, but the reduced intensities of a coincifence measurement result in uncertainties and ami iguities, especially for weak transitions. Despite major efforts at TRISTAN, described by Walters, many spins and multipolarities remain undetermined. In fact, in many cases even singles NO measurenents are unable to resolve all ambiguities in decay scheme studies, and of ten botn angular correlations and NO are required to do a complete analys is ([15], 
[16]). Hamilton [17] earlier and Wood at this Symposium have emphasized the crucia? importance of complementary data in order to determine EO amplitudes. Such data are important because $\mathrm{OO}$ admixtures can provide unique signatures of shape changes between two bands in a nucleus. Internal conversion data for mixed $E O+M 1+E 2$ transitions cannot give the EO admixtures directly, unless one has independent information (such as from $N O$ ) on the $M 1+E 2$ amplitudes. Indeed, in some cases one will not be able to establish that an EO admixture is even present without an E2/MI mixing ratio when MI internal conversion coefficients are much larger that E2 as in higher $Z$ nuclei. Hood has also emphasized how NO data will be essential in identifying the low-lying $0^{+}$ "intruder" states.

The importance of having good daia on nuclear moments in testing nuclear models has been emphasized in the summaries given at the Symposium by Krane and by Berkes. Speidel has shown how measurements of magnetic dipole moments of highly excited states are essential in understanding the structure that contributes to the backbend of the moment of inertia, and Heyde has shown how IBA-2 has recently achieved considerable success in calculating the moments of the first excited collective $2^{+}$states, in which the 9 -factors are systematically smaller than the collective value $Z / A$. The precise measurement of magnetic dipole moments, especialily by NMR/ON, has the potential to offer insight into the structure of the decaying ground states (as for example the Leuven work on the In isotopes referenced above). The use of atomic beam magnetic resonance and laser spectroscopy to measure magnetic moments far from stability will certainly complement future NO work (see the review of ISOLDE work by Ekström).

Quadrupole moments have a similar importance in nuclear structure. Hagn has summarized for us the two principal No measuring techniques (NMR/ON subresonances in ferromagnets and quadrupole alignment in crystals), and Meyer has discussed the need to know the quadrupole moment in order to determine the magnitude and sign of the deformation in the new light deformed regions far from stability. As examples of the importance of knowing the magnitude and sign of the quadrupole moment, it is essential to the development of our theoretical understandings of shape coexistence in the light $\mathrm{Hg}$ nuclei to measure especially the signs of the quadrupole moments of the two shapes in these nuclei. Some theories predict the same signs and others opposite signs for these shapes. The same is true in other regions such as the $M=Z=38$ region where prolate and oblate shapes are predicted, and in the $Z=38-40, N=59$ region.

Finally, in the case of E2/MI multipole mixing ratios, we have learned from Rikovska how IBA-2 has the potential to yield branching ratios and mixing ratios that can be compared with NO results (for example, the recent Daresbury on-line work on Te isotopes). The work reported here by Marshak demonstrates the high precision to which NO can determine E2/M1 mixing ratios and how current theories cannot explain the variation in $\delta$ even in a "well-understood" nucleus like ${ }^{166}$ Er.

\section{Exciting orospects for new physics}

Studies of nuclei far from the valley of beta stability have made major impacts on our understandings of the shapes and structure of nuclei. As an example, the $\mathrm{Sr}$ nuclei have been traced from $385 \mathrm{Sr}_{40}$ to $1805 \mathrm{Sr}_{62}$ (over $50 \%$ change in $N$ from 40 to 62 ), Fig. 1 . The trace of the $2^{+}$energies alone provides much information as it illustrates the smooth increase in the deformation of these nuclei from one new region of very strong deformation at $N=38(B=0.35)$ [18] to a spherical structure at $N=50$; then, unlike the lower side, they remain near spherical out to $N=58$ and suddeniy go to strongly deformed shapes at $N=$ 60 i $\mathrm{B}=0.35$ ). Clearly there are important differences in the structures of these nuclei to either side of $N=50$ which lead to this quite marked difference 


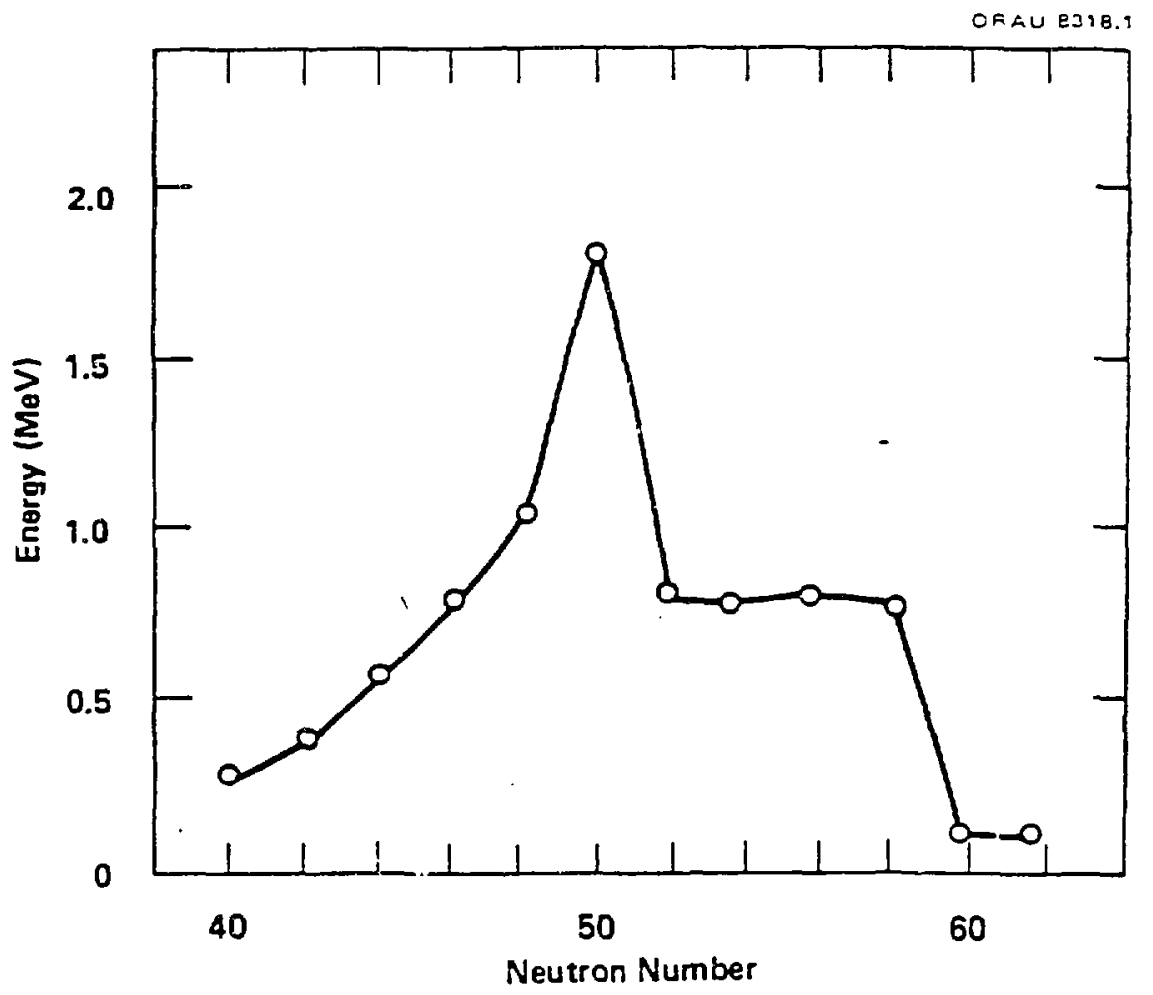

Figure 1. Energy levels of first $2^{+}$states of even-even Sr nuclei.

in the way deformation is approached. The difference in the two regions is related to the strong, very local reinforcing of spherical shell gaps at $N=56$ and $Z=40$ in addition to the stronger reinforcement at $N=50, Z=40$ to give spherical shapes as in double closed shell $\$ 8 Z \mathrm{Zr}_{50}$. The $N=56, Z=40$ reinforcement keeps the $\mathrm{Sr}$ nuclei with $N>50$ spherical longer until there is a switch in importance to different shell gaps where the $Z=38, N=60$ gaps at large deformation reinforce each other to suddenly override the spherical gaps as pointed out by Hamilton et al. [19] The very large jeformation in the very i iant $\mathrm{Sr}$ isotopes result $\mathrm{S}$ from the reinforcement of the $N=38$ and $Z=38$ shei? gaps at large deformation. Such studies of long isotapic and isotonic sequences of nuclei place new and stringent demands on theoretical nuclear models since structures characteristic of all the individual models developed for specific regions, from harmonic cscillator through triaxial rotor to rigid axially symetric rotor, may be found in one sequence. Thus, models such as the generalized collective model developed by the Frankfurt group (see Seiwert et a.. [20] and references therein) based on a description of collective states as quadrupole surface excitations and the Interacting Boson Model (IBA) developed by Arima and Iachello [21] with its different symmetries have been particularly attractive because they can track such changes over long sequences. These two approaches have been shown to be equivalent descriptions, with the beauty of IBA lying in its mathematical elegance, and the beauty of the generalized collective mode $i$ in its vivid physical pictures. Below we give a few illustrations where NO studies can provide crucial data for testing nuclear models.

In evei-even nuclei far off stability, we have already gone in many regions about as far off stability as we can in establishing level spins and parities by using some hard data and some soft data combined with systematics, particularly if more than the first one or two levels are of interest. Knowledge of only the $2_{1}^{+}, 2_{2}^{+}$, and $4_{1}^{+}$states can yield significant insights 


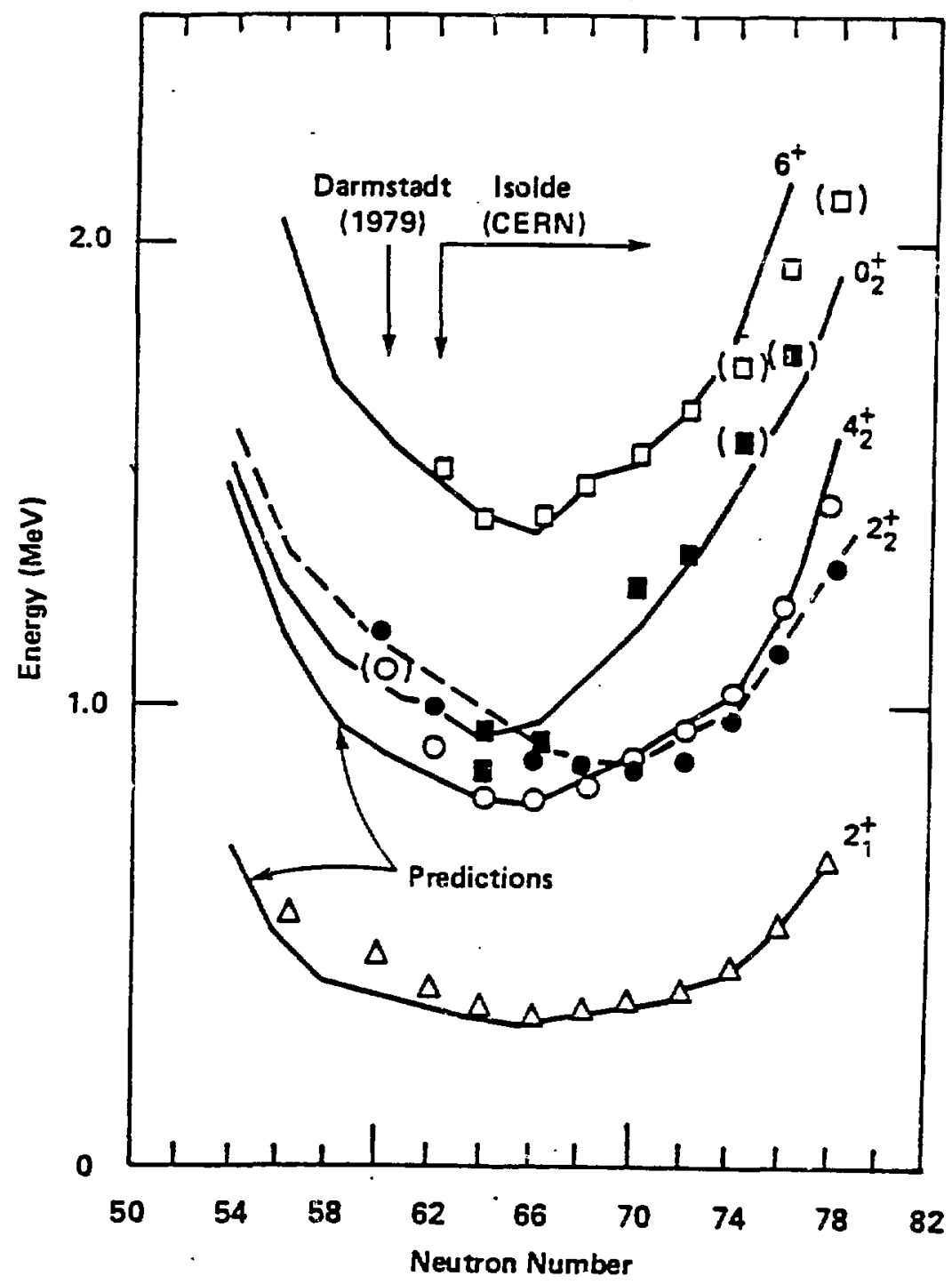

Figure 2. IBA calculations for even-even $X e$ isotopes compared with experimental data.

in the changes in structure as emphasized by Iachello [20] and illustrated in Figure 2 for $X e$ isotopes. The beauty of No with dilution refrigerators is that because the data are collected in singles mode, one can quickly acquire the data to establish the spins of the first few states. The competing technique of conversion coefficient measurements is useful only in higher $Z$ nuclei where ICC's are larger. Even there, ambiguities can occur since an E2 transition to the $2_{1}^{+}$level can come from a state of spin $0,2,3$ or 4 . The other competing technique of $\gamma-\gamma(\theta)$ cannot be used in far off stability nuclei produced with heavy ions and is useful for only the strongly populated levels when produced by other techniques. Thus, No data (or complementary in-beam $y$-ray angular distributions) go from easier (with less accelerator time to collect the data) and more definitive, to essential as $Z$ decreases. 


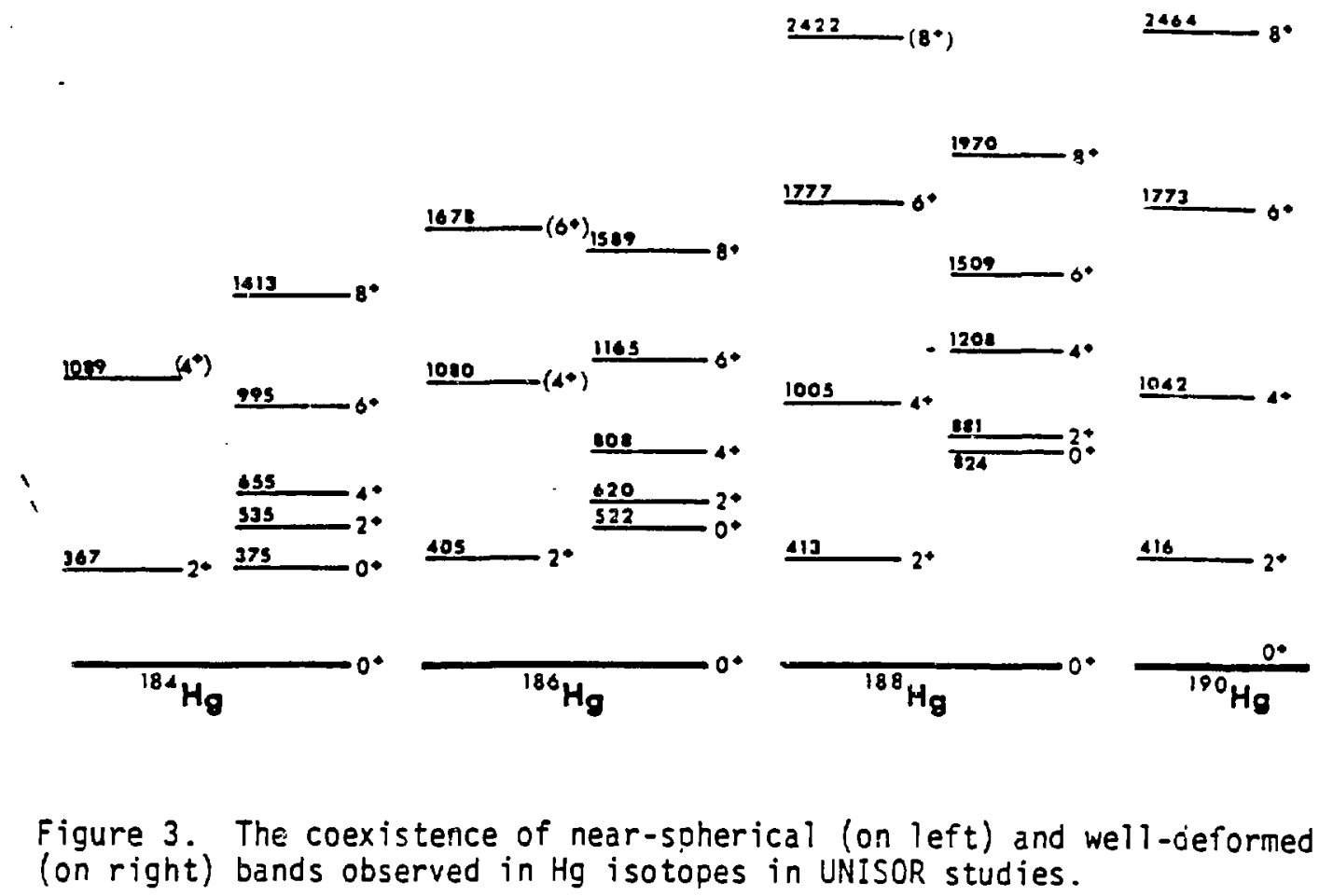

(on right) bands observed in $\mathrm{Hg}$ isotopes in UNISOR studies.

Beyond the above considerations, in-beam data may reveal only the yrast cascade states, and so other important states at low and higher spin from other bands may be missed. Moreover, conversion coefficient data even at high $Z$ may not yield assignments of important states. Figure 3 shows the systematics of the light mass mercury isotopes which provide classic examples of shape coexistence in nuclei. Note the two separate bands of levels built on quite different shapes, a near spherical ground state band $(|B|-0.12)$ and a welldeformed excited band $(B-0.25)$. These nuclei illustrate both of the points just made. The $0_{2}^{+}, 2_{2}^{+}, 4_{2}^{+}, 6_{2}^{+}, 8_{2}^{+}$states were not seen in any of the initial in-beam studies nor were they seen in the recent in-beam studies in $182 \mathrm{Hg}$. In the decay studies of 184-18871 to $184-138 \mathrm{Hg}$ (Hamilton et al. [23], Cole et al. [24]) conversion coefficients could not be obtained to establish the $4_{2}^{+}, \bar{\sigma}_{2}^{+}$, and $8_{2}^{+}$assignments in several cases. Those assignments in parentheses are based on energy systematics with the heavier $\mathrm{Hg}$ isotopes. These spins could be easily established by NO studies. Similarly, in other regions systematics have been used but clearly one is on dangerous ground to continue further off stability with the use of systematics to assign spins.

Take another example from the 1 ight $Z$ region, ${ }^{76} \mathrm{Kr}$. This was an essential nucleus in the discovery of a new region of strong deformation centered around $N=Z=38$ (Piercey et al. [25]). One of the critical data was the low lying $0^{+}$state at $770 \mathrm{keV}$. $80 \mathrm{th}$ in-beam studies and on-line decay studies of separated ${ }^{7} 6_{\mathrm{Rb}}$ were carried out. While the 0 spin was finally established from in-beam $\gamma$-ray angular distrib'tions in subsequent runs, with No data this would have easily been known one to two years earlier from the decay studies along with the spins of the levels that feed this state. 
Another important question to be answered in this region is where the $0^{+}$ spherical states are in the light strontium nuclei. In-beam data have yielded no evidence for such. Radioactive decay studies with No should be able to locate these states, which will be important in testing the ability of our theoretical models to explain this region. Much attention is devoted today to understanding how nuclei behave at high angular momentum. Information on the energies of the band heads of both the spherical and deformed structures in all these nuclei around $A=64-84$ will be essential in understanding their high spin structures as well. Already Hamilton et al. [26] have noted the marked differences between $78-82 \mathrm{Sr}$ and $74-78 \mathrm{Kr}$ nuclei at spins of $10^{+}-18^{+}$. At still higher spins, 20-30 h, both these shapes may be important in the yrast spectrum in different ways as multiple pairs of quasi-particles rotation align with the different available cores. Thus, again low spin information obtainable with NO measurements will be crucial. In the heavy Sr nuclei where large deformation was discovered, NO studies could establish several important spins which are still not known and which bear on the proper interpretation of low-spin deformed rotational structure, for example, ${ }^{98} \mathrm{Sr}$ shown in Figure 4 .

Wood at this Symposium has discussed the importance of NO data for odd-A nuclei in the Pt-Au-Ti-Hg region. Similar arguments $\mathrm{Can}$ be made throughout the periodic table. Of particular importance is the establishment of the ground state spins in these nuciti. Another crucial region, for example, is again the light $\mathrm{Kr}$ isotopes. The single particle energy levels of Bengtsson et ai. [27], calculated with a folded-Yukawa single particle potential, show gaps at $N=Z=38$,

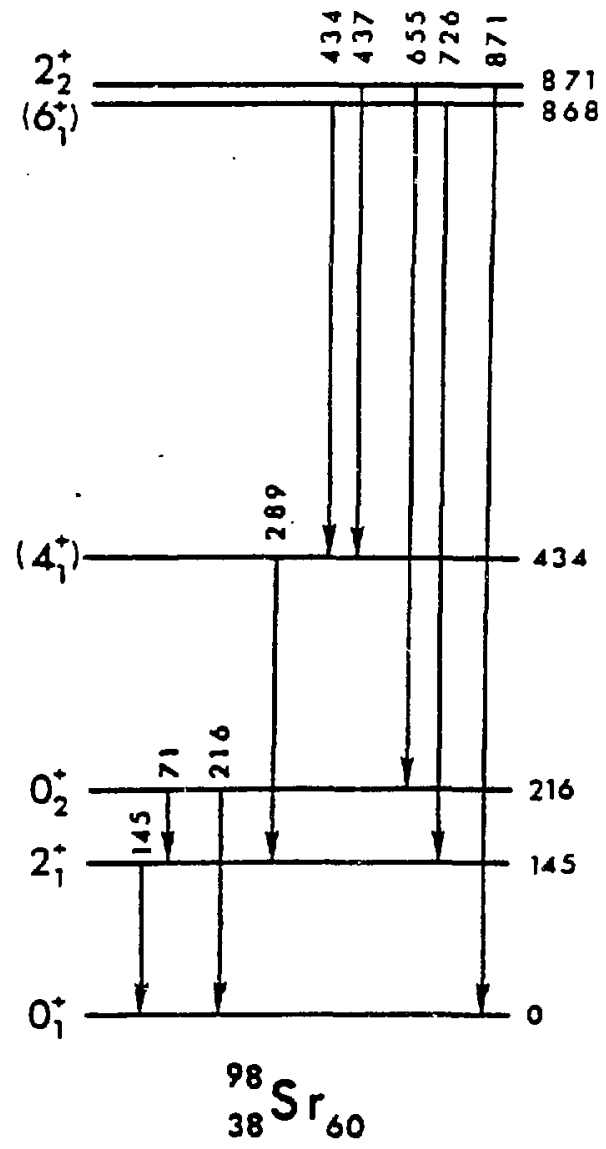

Figure 4. The low-iying level structure of ${ }^{98} \mathrm{Sr}$. 
for large prolate deformation and at $N=Z=36$ for large oblate deiormation. The puzzling theoretical question is why have these large oblate deformations not been seen in the light $\mathrm{Kr}$ or Se nuclei. In-beam studies of $75 \mathrm{Kr}$ have been used to show that $75 \mathrm{Kr}$ has a large prolate deformation [28]; however, the band structure in $75 \mathrm{Kr}$ as shown in Fig. 5 depends on arguments primarily from systematics to assign the spin of the ground state. From NMR/ON studies of $75 \mathrm{Kr}$, it should be possible to measure this spin and so firmly establish the data. A similar example is found in ${ }^{73} \mathrm{Se}$ where the ground state spin is unknown. $A$. Vanderbilt-Florida-LSU-UNISOR-Rochester collaboration is looking to identify levels in ${ }^{73} \mathrm{Kr}$, which should have a strong cblate deformation if recent theories are correct. The use of systematics here will be more dangerous in assigning the ground state spins.

Scrong deformation ( $B-0.35$ ) has aiready been reported in odd-A ${ }^{99} \mathrm{Sr}$, , $99,10 \mathrm{l} \mathrm{Sr}$ and $10 \mathrm{l} \mathrm{Zr}$ (Wohn et al. [29]). However, in every case the band head spins are not known. One must have at least one band head spin to definitively establish these bands for comparison with theory. Again, NMR/ON should be useful in such studies as well as helping to establish the higher spins.
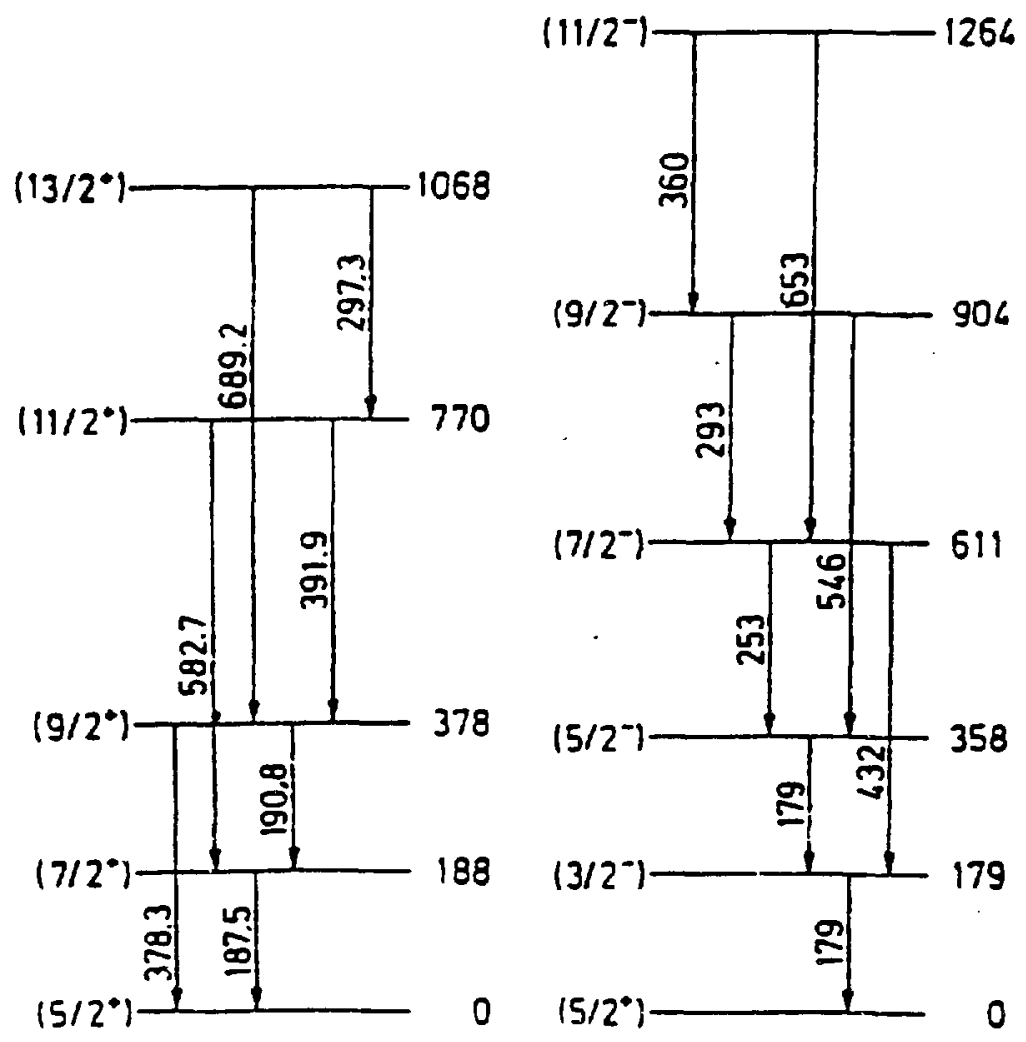

${ }^{75} \mathrm{Kr}$

Figure 5. Rotational band structure in ${ }^{75} \mathrm{Kr}$. 
Theoretical calculations have reached new levels of sophistication so that odd-odd nuclei are becoming important testing grounds of nuclear models as noted by Faessler [30]. Moreover, thase isotopes often have isomers whose locations are important for the theoretical calculations. The spins of trese isomers are essential. It is currently meaningful to start investigating the nature of the multiplets $i$ : odd-odd transitional nuclei, in order to learn about the specific interactions, couplings and coupling schemes in these nuclei and ultimately to unify the understanding of spherical multiplets and deformed Gallagher-Moszkowski doublets. Moreover, odd-odd isoiopes of ten have isomers whose locations and properties are important in many ways. One current example is in high-spin spectroscopy, where unique information can be obtained from the rotational signature splitting in odd-odd nuclei provided that the structure of the band head is known. The orientation facility will be crucial in all such studies of odd-odd nuclei where one has no assured starting spin and arguments rased on systematics are even more risky.

In many such cases, NO work will supply crucial results, including the ground state spin (by comparing $\mu B$ from NO angular distribution with gB from NMR/ON, the spin is measured directly), magnetic moments (essential to identify configurations, especially in odd-odd nuciei), and quadrupole moments (the most reliable and direct measure of the nuclear deformation).

The measurements of E2/M1 mixing ratios have long provided very sensitive tests of different nuclear models as detailed, for example, by Lange et al. [31] in their recent theoretical and experimental review of such measurements. We need not repeat the long history of significant contributions tu our theoretical understandings by such measurements. These E2/MI mixing ratios continue to be important in testing theories, particularly so in the generalized coilective model approach of potential energy surfaces and in IBA which are the best approaches to tracking the properties of long mass chains of nuciei.

One of the most important outgrowths of studying dynamical symmetry in collective nuclear structure physics is the search for dynamical supersymnetry. Now supersymmetry searches are being extended to nuclei far off stability. The concept of supersymmetry (SUSY) was proposed by high energy physicists some ten years ago in an attempt to unify all forces in nature. In recent years, the SUSY idea has been utilized in other aspects of physics as well (nuclear structure physics and polymer physics, to name a few). Nevertheless, it is fair to say that even though it is intellectually satisfying to have a unifying theoretical scheme, it is also true that there has been little empirical evidence for SUSY to support its validity. The situation is altered by the recent postulation by Iachello [32] that SUSY may also be applicable in the realm of collective structure in nuclei where one can obtain a wealth of data for empirical verification.

With nuclear orientation refrigerators on line with on-line isotope separators, one car not only extend the tests of SUSY to nuclei far off stability but equally as important one can measure the E2/MI branciing ratios down to even very weak ( $-1 \%$ intensity) transitions in complex decay schemes with several hundred transitions to provide crucial tests of SUSY predictions. often the E2 strengths of these weak transitions, which cannot be studied by conventional $\gamma-\gamma(\theta)$ directional correlation coincidence studies in complex decays far off stability, are the essential ones to know in order to test SUSY: Thus, an on-line NO refrigeracor is a powerful, new tool to provide crucial data to test nuclear models including SUSY, especially to track the structure across the multiplet. Such a search for evidence for suSY farther off stability in l'9lpt has been initiated by a UNISOR-Leuven collaboration with the on-line refrigerator at Leuven to measure E2/M1 mixing ratios and spins to complete UNISOR level scheme studies. Suffice it to say here that to test supersymmetry, one needs accurate spins for al? the levels. While the energies and spins and 
parities of the levels provide major tests, the E2 strengths of all transitions are among the important predictions of supersymmetry. Even though supersymmetry is expected in many areas of physics, nuclear physics seems to be the only realm where SUSY may be lurking in an (effective) manner that one can test the theory empirically and NO facilities can play an important role.

Other very important data for testing nuclear models are E0/E2 ratios. Again, Lange et al. [31] have reviewed the importance of such data and previous tests. Electric monopole, EO, radiation is particularly important because it arises from the penetration of the atomic electrons into the nuclear volume. Because the monopole matrix element is proportional to the square of the nuclear radius, it is a sensitive test of nuclear shape changes, and this is well established experimentally [31].

In the UNISOR study [23], [24] of the $0_{2}^{+}$'band heads and first $2^{+}$members of prolate deformed bands and the subsequent establishment of shape coexistence in $184,186,188 \mathrm{Hg}$, the EO transitions from the $\mathrm{O}_{2}^{+}$and $2^{+}$levels were of prime importance. Further tests of the theoretical models could be made in these nuclei if the E2/MI ratios in the $2_{\text {def }}^{+}+2_{\text {sph }}^{+}$and $4_{\text {def }}^{+} \rightarrow 4_{\text {sph }}^{+}$transitions were known so the EO/E2 ratios could be extracted.

Zganjar et al. [33] used EO admixtures as signatures to identify bands in ${ }^{187} \mathrm{Au}$ coupled to the $\mathrm{O}_{1}^{+}$and $\mathrm{O}_{2}^{+} \mathrm{Pt}$ cores with different deformations. The conversion coefficients were sufficiently large to indicate definite EO admixtures there. In a similar search in $189 \mathrm{Tl}$, candidates for coupling to both the $\mathrm{O}_{1}^{+}$and $\mathrm{O}_{2}^{+}$states in $188_{\mathrm{Hg}}$ with different deformation were not found, although transitions with essentially pure MI conversion coefficients were found. Without measuring the E2/MI mixing ratios it is impossible to tell if these transitions are EO/E2 or MI. Such cases may occur in other nuclei in this and other regions. Thus, NO measurements are essential in this region to extend our understanding of shape coexistence by distinguishing EO/E2 transitions from MI or E2/MI transitions. As well, one would simultaneous ly measure the spins of all the band members.

\section{Future prospects and applications}

4.1 Multiparameter analysis. By this is meant the acquisition of sufficient spectroscopic information in order to extract spins and multipolarities for each $Y-r$ ay in the decay. Present schemes for measuring $W\left(0^{\circ}\right) / W\left(90^{\circ}\right)$ do not give sufficient information to yield the desired nuclear parameters. Possible sources of the additional information include the following: (1) development of reliable schemes for normalizing to the variations in implantation rate between coid and warm data, which will allow independent determination of $W\left(0^{\circ}\right)$ and $W\left(90^{\circ}\right)$, and thus of the $P_{2}$ and $P_{4}$ terms in the angular distribution; (2) gama-gamma angular correlations, not only for help in elucidating the properties of single garna rays, but also for the possibility that the ry correlation holds the key to the analysis of all of the NO data; (3) linear polarization measurements on the $y$-rays from in oriented source, which can complement the ordinary No angular distributions in providing unambiguous analysis of decay schemes including the relative parity of nuclear states; (4) internal conversion coefficients, which are often of limited value, for example in distinguishing M1 from E2 transitions in middle-weight nuclei, but which may be useful in permitting the deduction of relative parities.

4.2 Relaxation and pre-orientation. In general, as one moves further from stability, the half-lives become shorter. As has already been mentioned above, for decay half-lives below about one minute, there is likely to be reduced ori- 
entation because of incomplete relaxation of the implanted ion to its polarized environment. This effect is of course temperature dependent, so that the lower one goes in temperature in attempting to increase orientations, the longer the relaxation times become, resulting in the possibility of a decrease in orientation. To solve this problem, the Leuven group have proposed a pre-orientation stage, in which the ion beam is reflected at grazing incidence from a magnetized single crystal of nickel [7]. The function of the implantation target would then be primarily to maintain the orientation acquired in the scattering. It is expected that the range of half-lives $c$ an be thus extended to as short as one second. Pre-orientation by lasers is also a possibility.

4.3 New separators. A recent Vanderbilt-LSU-Florida-UNISOR-Rochester collaboration has used a recoil mass spectrometer to do in-bean $r$-speciroscopy studies of $f$ ar off stability nuclei in the $A=70-80$ region. In particular, the studies seek to identify ${ }^{73} \mathrm{Kr}$ to search for theoreticaliy predicted oblate deformation. They have shown that high qualit; in-beam data can be obtained from recoil-particle- $\gamma$ and $\gamma-\gamma$ coincidences. Since in the recoil mass spectrometer or velocity filter one has no limitation on haif life (down to $10^{-6}$ s) or element, this is a universal system. A NO facility on line with either or both of these devices will open up studies not possible with conventional on-line isotope separators. Such a. future facility will extend again our abjlity to map nuclear structures in $N$ and $Z$.

4.4 Alpha decay. The decays of many ground states in heavy nuclei go largelv by alpha emission. The Leuven group (Vandeplassche et al. at this Symposium) have made preliminary tests with alpha detectors in the $\overline{i r}$ cryostat and are optimistic about the development of the technique for this region of nuclei. Very little systematic work has been done on alpha decay by NO, and this development could open up new avenues for nuclear structure studies.

\section{Acknowledgements}

It is with great appreciation that we thank the speakers at this Symposium for the quality of their efforts, the contributors of papers for providing us with the benefits of their labors, and the many experts who shared their wisdom at discussions formal and informal.

Special gratitude must go to L. Vanneste, for organizing such an excellent and stimulating program, and to $O$. Vandeplassche, $E$. Van Walle and $J$. Wouters for keeping things running smoothly. Finally, we extend our thanks to those early feminists of the 17 th century who had the foresight to provide us with such a lovely setting for our discussions.

This work was partially supported by the U.S. Department of Energy under contracts DE-ATO6-83ER40109 (K.S.K.) and DE-ASO5-76ER05034 (J.H.H.).

\section{References}

[1] H. Postma and N.J. Stone in Low-Temperature Nuclear Orientation (Amsterdam. North-Holl and, 1985), Chapter 1 .

[2] C.S. Wu, E. Ambler, R W. Hayward, D.D. Hoppes and R.P. Hudson, Phys. Rev. 105 (1957) 1413.

[3] P.6. Hansen, Ann. Rey. Nucl. Part. Sci. 29 i1979) 69.

[4] J.H. Hamilton, P.G. Hansen and E.F. Zganjar, Reports on Progress in Physics, to be published.

[5] Oxford Instruments Ltd.; Gsney Mead, Oxford 0x2; Oox, England. 
[б] P. Herzog, i. -R. Folie, K. Freitag. A. Kluge, M. Reuschenbach, and E. Bodenstedt, Nucl. Inst. Meth. 155 (1978) 421.

[7] D. Vandeplassche, L. Vanneste, H. Pattyn, J. Geenen, C. Nuytten and E. yan Walle, Nucl. Inst. Meth. 186 (1981) 211.

[8] N.J. Stone and W.O. Hamilton, Hyp. Int. 10 (1981) 1219; V.R. Green. H.D. Hamilton, S.J. Robinson, N.J. Stone and P.M. Walker, Hyp. Int. 15/16 (1983) 979; W. Gelletly, Nucl. Inst. Meth. 211 (1983) 89.

[9] P. Herzog, K. Freitag, C. Herrman, and K. Schlösser, Hyp. Int. 15/16 (1983) 329.

[10] D. Vandeplassche, E. yan Halle, C. Nuytten and L. Vänneste, Phys. Rev. Lett. 49 (1982) 1390.

[11] D. Vandeplassche, E. van Walle, C. Nuytten, and L. Vanneste, Nucl. Phys. A396 (1983) 115c.

[12] C. Nuytten, D. Vandeplasscte, E. van Walle and L. Vanneste, Phys. Rev. C 26 (1982) 1701.

[13] C. Nuytten, D. Vandeplassche, E. van Walle and L. Vanneste, Phys. Lett. $92 \mathrm{~A}$ (1982) 139.

[14] C. Nuytten, D. Vandeplassche, E. van Walle and L. Vanneste, 2. Phys. 50 (1983) 51.

[15] W.M. Lattimer, K.S. Krane, N.J. Stone and G.Eska, J. Phys. G 7 (1981) 1713.

[16] H.H. Ghaleb and K.S. Krane, Nucl. Phys. A 426 (1984) 20.

[17] J.H. Hamilton, in Heavy Ion Collisions, ed. by R. Bock (Amsterdam, NorthHolland, 1982), vol. 3, P. 574 .

[18] R.B. Piercey, A.V. Ramayya, J.H. Hamilton, X.-J. Sun, Z.Z. Zhao. R.L. Robinson, H.J. Kim and J.C. Wells, Phys. Rev, C25 (1982) 1914.

[19] J.H. Hamilton et al., J. Phys. G 10 (1984) L87.

[20] M. Seiwert, J.A. Maruhn. and W. Greiner. in High Angular Momentum Properties of Nuclei, ed. by N.R. Johnson (New York, Harwood, 1982), P. 249.

[21] A. Arima and F. Iachello, Ann. Rev. Nucl. Sci. 31 (1981) 75.

[22] F. Iachello, in Future Directions in Studies of Nuclei Far From Stability, ed. by J.H. Hamitton et al. (Amsterdam, North-Holland. I980), P. 281 .

[23] J.H. Hamilton et al., Phys. Rev. Lett. 35 (1975) 562.

[24] J.D. Cole et al., Phys. Rev. Lett. 37 (1976) 1185.

[25] R.B. Piercey et al., Phys. Rev. Lett. 47 (1981) 1514.

[26] J.H. Hamilton et al., in High Angular Momentum Properties of Nuclei, ed. by N.R. Johnson (New York, Harwood, 1982), p. 227.

[27] R. Bengtsson, P. Möller, J.R. Nix and J.-Y. Zhang, Physica Scripta 29 (1984) 402.

[28] M.A. Herath-Banda, A.Y. Ramayya, G. Leander and J. Eberth, to be published.

[29] F.K. Wohn, J.C. Hill, R.F. Petry, H. Dejbakhsh, Z. Berant and R.L. Gill, Phys. Rev. Lett. 51 (1983) 873.

[30] A. Faessler, in Future Directions in Studies of Nuclei Far Frcm Stability; ed. by J.H. Hamition et al. (Amsterdam, North-Holland, 1980), P. 1.

[31] J. Lange, K. Kumar and J.H. Hamilton, Rev. Mod. Ph. 54 (1982) 119.

[32] F. Iachello, Phys. Rev. Lett. 44 (1980) 772.

[33] E.F. Zganjar, J.D. Cole, J.L. Wood and M.A. Grimm, in IV Int. Conf. on Nuclei Far From Stability, CERN 81-09 (1981), p. 630. 
PROC. 1964 FALL MEETING OF DIUISION OF NUCLEAR FHYSICS

oct. 16-20, 1904, iashmile, TN

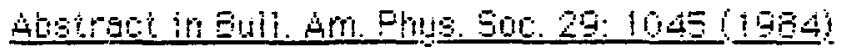

Hyperfine structures and isotone Shifts of $201.203 .205 \mathrm{Ti}$ oy

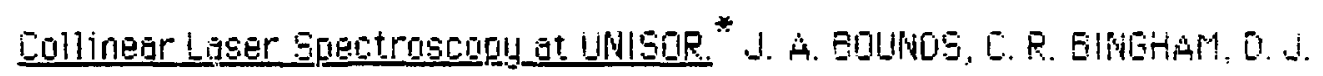

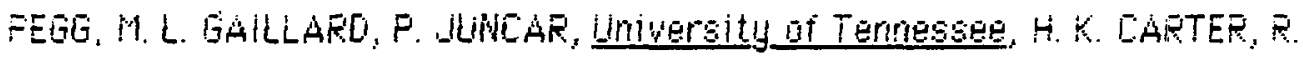

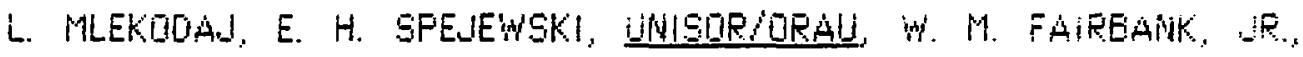

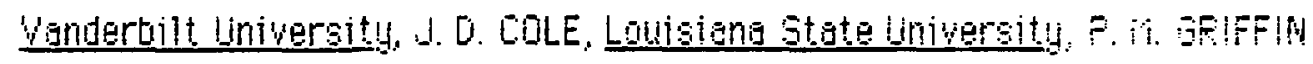

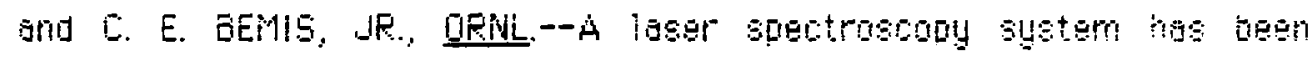
developed to abserve hyperfine structure and isotope shite af mose-seported thailitum stoms. Utilizing beams of stoble -02205 Th sod

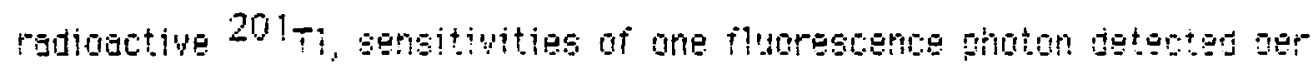

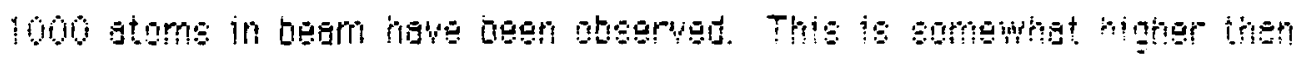

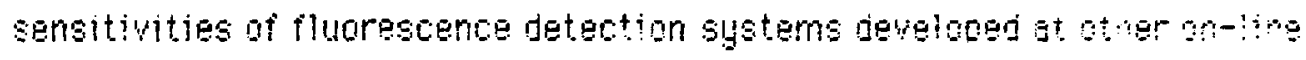

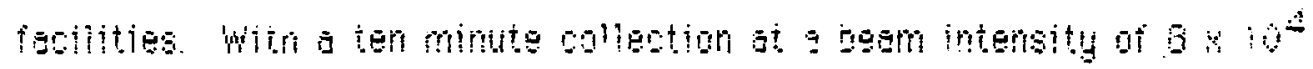
$\sec ^{-1}$ spectrum of $201_{T}$ suficient to deduce HFs and 15 was otched. These results indicate that these studies can be performed an any thallum isotoe for which at iegst $6 \times 10^{4}$ atoms por socond of goorate begm current sre sualioble.

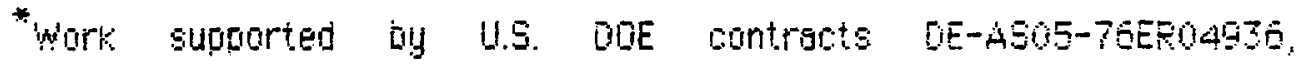
DE-ACO5-760R00033, DE-A505-76EP05034, DE-AS05-76ER04935, Ind $D E-A C 05-640 R 2 ! 400$. 